CRYSTALLOGRAPHIC COMMUNICATIONS

ISSN 2056-9890

Received 13 April 2017

Accepted 19 April 2017

Edited by H. Stoeckli-Evans, University of Neuchâtel, Switzerland

Keywords: crystal structure; pharmacopoeia reference standard; hydrogen bonding..

CCDC reference: 1544853

Supporting information: this article has supporting information at journals.iucr.org/e

\section{Crystal structure of isopropyl 2-hydroxy-2-phenyl- acetate: a pharmacopoeia reference standard}

\author{
Ivan Isaiev, ${ }^{\mathrm{a} *}$ Svitlana Shishkina, ${ }^{\mathrm{b}}$ Igor Ukrainets $^{\mathrm{c}}$ and Elena Bevz ${ }^{\mathrm{c}}$
}

${ }^{\text {a}}$ V. N. Karazin Kharkiv National University, 4 Svobody Sq., Kharkiv 61077, Ukraine, 'bSSI 'Institute for Single Crystals', National Academy of Sciences of Ukraine, 60 Nauky Ave., Kharkiv 61001, Ukraine, and 'National University of Pharmacy, 4 Valentinivska St., Kharkiv 61168, Ukraine. *Correspondence e-mail: swhtlover@gmail.com

The title compound, $\mathrm{C}_{11} \mathrm{H}_{14} \mathrm{O}_{3}$, is used as a pharmacopoeia reference standard for determining impurities in the drug Pregabalin, used for the treatment of epilepsy and diabetic neuropathic pain. The molecule is far from being planar, with the dihedral angle between the planes of the aromatic ring and the carboxyl fragment $(\mathrm{O}-\mathrm{C}=\mathrm{O})$ being $76.1(6)^{\circ}$. The isopropyl substituent is located in a synperiplanar position relative to the $\mathrm{C}=\mathrm{O}$ bond and is turned so that the $\mathrm{C}-$ $\mathrm{O}-\mathrm{C}-\mathrm{H}$ torsion angle is $-43.7^{\circ}$. In the crystal, bifurcated $\mathrm{O}-\mathrm{H} \cdots(\mathrm{O}, \mathrm{O})$ hydrogen bonds, enclosing $R_{1}^{2}(5)$ ring motifs, lead to the formation of chains propagating along the $c$-axis direction. Inversion-related chains are linked by the $\mathrm{C}-\mathrm{H} \cdots \mathrm{O}$ hydrogen bonds, forming undulating layers lying parallel to the $b c$ plane.

\section{Chemical context}

Pharmacopoeia reference standards are used widely for identification and quantitative determination of an active ingredient and undesirable impurity contents in many drug substances (European Pharmacopoeia Supplement, 2017). The title compound is used as the pharmacopoeia reference standard for the determining the level of impurities in Pregabalin (European Pharmacopoeia Supplement, 2016). This drug, sold under the trade mark 'Lyrica' (Silverman, 2016) is used for the treatment of epilepsy and diabetic neuropathic pains. Until now, its molecular and crystal structure were unknown.

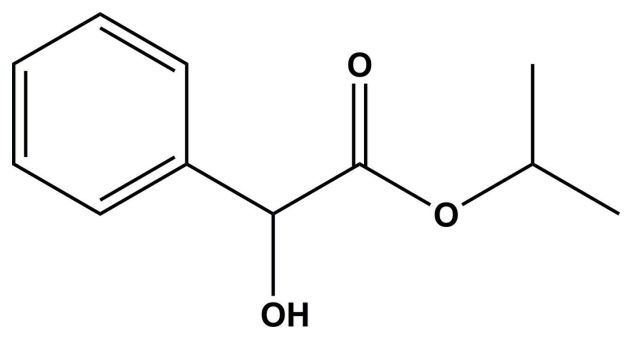

\section{Structural commentary}

The molecular structure of the title compound is shown in Fig. 1. The hydroxyl group is situated in the $-s c$ position relative to the $\mathrm{C} 1-\mathrm{C} 6$ endocyclic bond; torsion angle $\mathrm{C} 1-$ $\mathrm{C} 6-\mathrm{C} 7-\mathrm{O} 1$ being $\left.-46.2(6)^{\circ}\right)$. The ester substituent at atom $\mathrm{C} 7$ has a $+s c$-orientation with respect to bond $\mathrm{C} 1-\mathrm{C} 6$ bond, with torsion angle $\mathrm{C} 1-\mathrm{C} 6-\mathrm{C} 7-\mathrm{C} 8=71.2(6)^{\circ}$, and it is turned in such way that the dihedral angle between the planes 


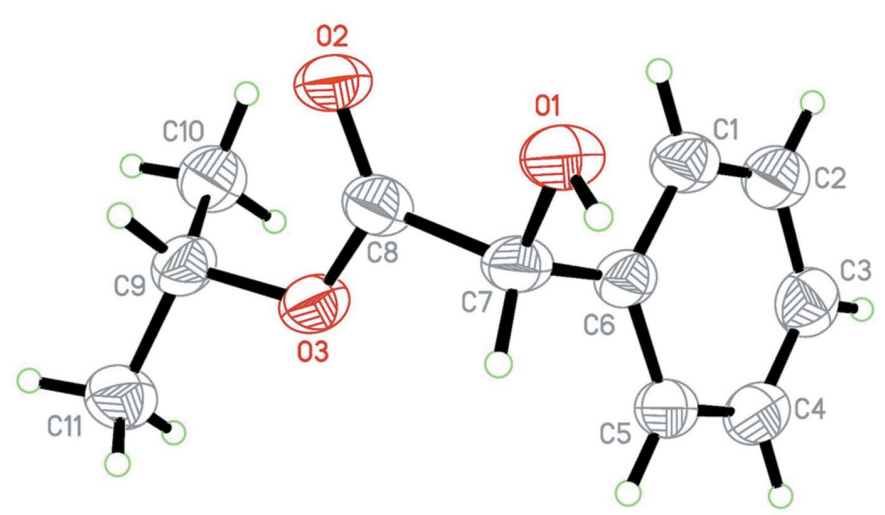

Figure 1

The molecular structure of the title compound, showing the atom labelling. Displacement ellipsoids are drawn at the $50 \%$ probability level.

of the aromatic ring $(\mathrm{C} 1-\mathrm{C} 6)$ and the carboxyl fragment (O3$\mathrm{C} 8=\mathrm{O} 2)$ is $76.1(6)^{\circ}$. The isopropyl substituent is located in a syn-periplanar position relative to the $\mathrm{C} 8=\mathrm{O} 2$ bond and is turned so that the $\mathrm{C} 8-\mathrm{O} 3-\mathrm{C} 9-\mathrm{H} 9$ torsion angle is $-43.7^{\circ}$.

\section{Supramolecular features}

In the crystal, molecules are linked by bifurcated $\mathrm{O}-$ $\mathrm{H} \cdots(\mathrm{O}, \mathrm{O})$ hydrogen bonds, forming chains propagating along [001] and enclosing $R_{1}^{2}(5)$ ring motifs (Fig. 2 and Table 1). Neighbouring chains are linked by $\mathrm{C}-\mathrm{H} \cdots \mathrm{O}$ hydrogen bonds, forming undulating layers lying parallel to the $b c$ plane (Table 1 and Fig. 3).

\section{Database survey}

A search in the Cambridge Structural Database (Version 5.38, update February 2017; Groom et al., 2016) for substructure

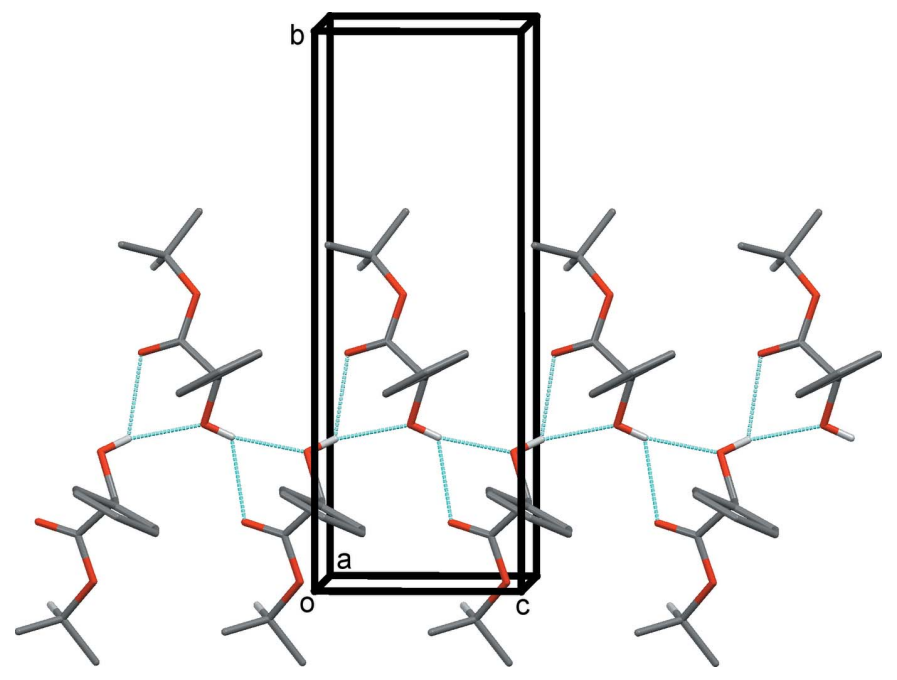

Figure 2

A partial view along the $a$ axis of the crystal packing of the title compound, with the hydrogen bonds shown as dashed lines (see Table 1). For clarity, only $\mathrm{H}$ atoms $\mathrm{H} 1 O$ and $\mathrm{H} 9$ have been included.
Table 1

Hydrogen-bond geometry $\left(\AA{ }^{\circ}\right)$.

\begin{tabular}{lllll}
\hline$D-\mathrm{H} \cdots A$ & $D-\mathrm{H}$ & $\mathrm{H} \cdots A$ & $D \cdots A$ & $D-\mathrm{H} \cdots A$ \\
\hline $\mathrm{O} 1-\mathrm{H} 1 O \cdots \mathrm{O} 1^{\mathrm{i}}$ & $0.83(6)$ & $2.12(5)$ & $2.903(2)$ & $158(5)$ \\
$\mathrm{O} 1-\mathrm{H} 1 O \cdots 2^{\mathrm{i}}$ & $0.83(6)$ & $2.38(6)$ & $2.930(5)$ & $124(5)$ \\
$\mathrm{C} 9-\mathrm{H} 9 \cdots 2^{\mathrm{ii}}$ & 1.00 & 2.53 & $3.379(7)$ & 142 \\
\hline
\end{tabular}

Symmetry codes: (i) $x,-y+\frac{1}{2}, z+\frac{1}{2}$; (ii) $-x+2,-y+1,-z$.

isopropyl 2-hydroxy-2-phenylacetate yielded three hits, viz. isopropyl 2,2-bis(4-bromophenyl)-2-hydroxyacetate (EFAFEY; Smith, 2012), 1-isopropyl 4-methyl 2-hydroxy-2-\{2[(methoxycarbonyl)amino]phenyl succinate (MAZJAA; Suárez-Castillo et al., 2012) and syn-isopropyl 2,3-dihydroxy-4methyl-2-phenylpentanoate (MERRIL; Scholtis et al., 2006). In the crystals of these three compounds, molecules are linked by pairs of $\mathrm{O}-\mathrm{H} \cdots \mathrm{O}$ hydrogen bonds, forming inversion dimers.

\section{Synthesis and crystallization}

To a solution of (2RS)-2-hydroxy-2-phenylacetic acid (15.22 g, $0.1 \mathrm{~mol}$; racemic mandelic acid) in $50 \mathrm{ml}$ propan-2-ol was added $0.5 \mathrm{ml}$ of concentrated $\mathrm{H}_{2} \mathrm{SO}_{4}$, and the mixture was refluxed for $5 \mathrm{~h}$ (Fig. 4). The excess of propan-2-ol was removed in vacuo. The reaction mixture was diluted with cold water and $\mathrm{Na}_{2} \mathrm{CO}_{3}$ was added to adjust the $\mathrm{pH}$ to 8 . The solution was extract with $\mathrm{CH}_{2} \mathrm{Cl}_{2}(3 \times 30 \mathrm{ml})$. The organic layers were combined and the solvent extracted by distillation (at reduced pressure at the end). The residue was distilled in vacuo, and a fraction with a boiling point of 361-363 K/4 mm $\mathrm{Hg}$ was taken, and then left for several hours in the refrigerator at $c a 278 \mathrm{~K}$, giving finally the title compound as colourless needle-like crystals (yield of $17.67 \mathrm{~g}, 91 \%$; m.p. 306.9-307.3 K).

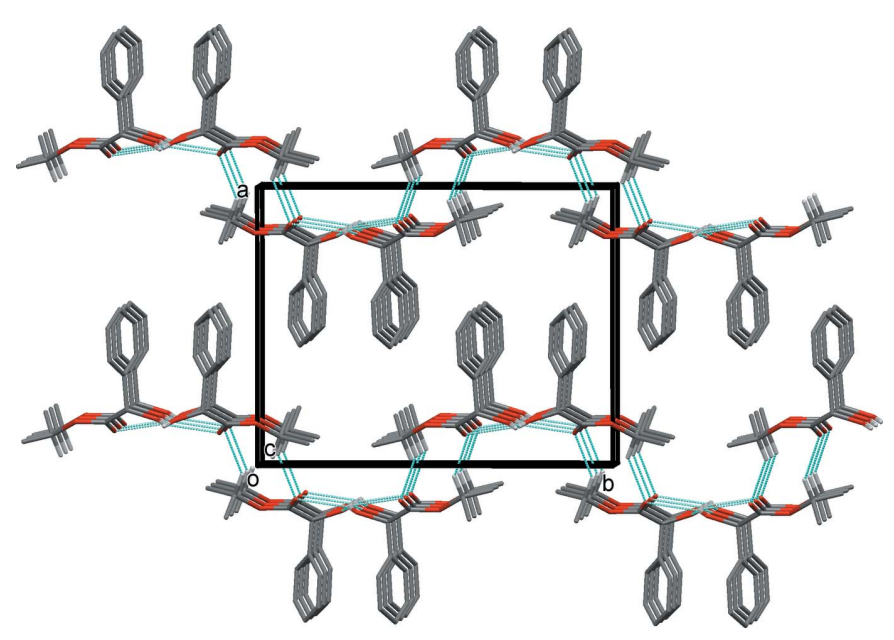

Figure 3

A view along the $c$ axis of the crystal packing of the title compound, with the hydrogen bonds shown as dashed lines (see Table 1). For clarity, only $\mathrm{H}$ atoms $\mathrm{H} 1 O$ and $\mathrm{H} 9$ have been included. 
<smiles>CC(C)[OH+]C(=O)C(O)c1ccccc1</smiles><smiles>[PH3+][SnH2]</smiles><smiles>CC(C)OC(=O)C(O)c1ccccc1</smiles>

Figure 4

Reaction scheme

\section{Refinement}

Crystal data, data collection and structure refinement details are summarized in Table 2. All of the $\mathrm{H}$ atoms could all be located from difference-Fourier maps. The hydroxyl $\mathrm{H}$ atom was refined with $U_{\text {iso }}(\mathrm{H})=1.5 U_{\text {eq }}(\mathrm{O})$. The C-bound $\mathrm{H}$ atoms were included in calculated positions and treated as riding: $\mathrm{C}-\mathrm{H}=0.93-0.97 \AA$, with $U_{\text {iso }}(\mathrm{H})=1.5 U_{\text {eq }}(\mathrm{C}$-methyl $)$ and $1.2 U_{\text {eq }}(\mathrm{C})$ for other $\mathrm{H}$ atoms.

\section{References}

Agilent (2012). CrysAlis PRO. Agilent Technologies, Yarnton, England.

European Pharmacopoeia Supplement (2016). pp. 5801-5803. Strasbourg: Council of Europe.

European Pharmacopoeia Supplement (2017). pp. 733-736. Strasbourg: Council of Europe.

Farrugia, L. J. (2012). J. Appl. Cryst. 45, 849-854.

Groom, C. R., Bruno, I. J., Lightfoot, M. P. \& Ward, S. C. (2016). Acta Cryst. B72, 171-179.

Macrae, C. F., Bruno, I. J., Chisholm, J. A., Edgington, P. R., McCabe, P., Pidcock, E., Rodriguez-Monge, L., Taylor, R., van de Streek, J. \& Wood, P. A. (2008). J. Appl. Cryst. 41, 466-470.

Scholtis, S., Ide, A. \& Mahrwald, R. (2006). Org. Lett. 8, 5353-5355.

Sheldrick, G. M. (2008). Acta Cryst. A64, 112-122.

Sheldrick, G. M. (2015). Acta Cryst. C71, 3-8.

Silverman, R. B. (2016). Technol. Innov. 17, 153-158.

Smith, G. (2012). Acta Cryst. E68, o3276.

Spek, A. L. (2009). Acta Cryst. D65, 148-155.
Table 2

Experimental details.

\begin{tabular}{|c|c|}
\hline \multicolumn{2}{|l|}{ Crystal data } \\
\hline Chemical formula & $\mathrm{C}_{11} \mathrm{H}_{14} \mathrm{O}_{3}$ \\
\hline$M_{\mathrm{r}}$ & 194.22 \\
\hline Crystal system, space group & Monoclinic, $P 2_{1} / c$ \\
\hline Temperature $(\mathrm{K})$ & 100 \\
\hline$a, b, c(\AA)$ & $11.872(3), 15.165(4), 5.6079(11)$ \\
\hline$\beta\left(^{\circ}\right)$ & $91.41(2)$ \\
\hline$V\left(\mathrm{~A}^{3}\right)$ & $1009.3(4)$ \\
\hline$Z$ & 4 \\
\hline Radiation type & Мо $K \alpha$ \\
\hline$\mu\left(\mathrm{mm}^{-1}\right)$ & 0.09 \\
\hline Crystal size $(\mathrm{mm})$ & $0.20 \times 0.08 \times 0.06$ \\
\hline \multicolumn{2}{|l|}{ Data collection } \\
\hline Diffractometer & Agilent Xcalibur Sapphire3 \\
\hline Absorption correction & $\begin{array}{l}\text { Multi-scan (CrysAlis RED; } \\
\quad \text { Agilent, 2012) }\end{array}$ \\
\hline$T_{\min }, T_{\max }$ & $0.357,1.000$ \\
\hline $\begin{array}{l}\text { No. of measured, independent and } \\
\text { observed }[I>2 \sigma(I)] \text { reflections }\end{array}$ & $5292,1761,922$ \\
\hline$R_{\text {int }}$ & 0.101 \\
\hline$(\sin \theta / \lambda)_{\max }\left(\AA^{-1}\right)$ & 0.594 \\
\hline \multicolumn{2}{|l|}{ Refinement } \\
\hline$R\left[F^{2}>2 \sigma\left(F^{2}\right)\right], w R\left(F^{2}\right), S$ & $0.090,0.255,1.03$ \\
\hline No. of reflections & 1761 \\
\hline No. of parameters & 132 \\
\hline $\mathrm{H}$-atom treatment & $\begin{array}{l}\mathrm{H} \text { atoms treated by a mixture of } \\
\text { independent and constrained } \\
\text { refinement }\end{array}$ \\
\hline$\Delta \rho_{\max }, \Delta \rho_{\min }\left(\mathrm{e} \AA^{-3}\right)$ & $0.30,-0.26$ \\
\hline
\end{tabular}

Computer programs: CrysAlis CCD and CrysAlis RED (Agilent, 2012), SHELXS2014 (Sheldrick, 2008), SHELXL2014 (Sheldrick, 2015), ORTEP-3 for Windows (Farrugia, 2012), Mercury (Macrae et al., 2008), PLATON (Spek, 2009) and publCIF (Westrip, 2010).

Suárez-Castillo, R., Bautista-Hernández, C. I., Sánchez-Zavala, M., Meléndez-Rodríguez, M., Sierra-Zenteno, A., Morales-Ríos, M. S. \& Joseph-Nathan, P. (2012). Heterocycles, 85, 2147-2171.

Westrip, S. P. (2010). J. Appl. Cryst. 43, 920-925. 


\section{supporting information}

Acta Cryst. (2017). E73, 771-773 [https://doi.org/10.1107/S2056989017005862]

Crystal structure of isopropyl 2-hydroxy-2-phenylacetate: a pharmacopoeia reference standard

Ivan Isaiev, Svitlana Shishkina, Igor Ukrainets and Elena Bevz

Computing details

Data collection: CrysAlis CCD (Agilent, 2012); cell refinement: CrysAlis CCD (Agilent, 2012); data reduction: CrysAlis RED (Agilent, 2012); program(s) used to solve structure: SHELXS2014 (Sheldrick, 2008); program(s) used to refine structure: SHELXL2014 (Sheldrick, 2015); molecular graphics: ORTEP-3 for Windows (Farrugia, 2012) and Mercury (Macrae et al., 2008); software used to prepare material for publication: SHELXL2014 (Sheldrick, 2015), PLATON (Spek, 2009) and publCIF (Westrip, 2010).

Isopropyl 2-hydroxy-2-phenylacetate

Crystal data

$\mathrm{C}_{11} \mathrm{H}_{14} \mathrm{O}_{3}$

$M_{r}=194.22$

Monoclinic, $P 2_{1} / c$

$a=11.872(3) \AA$

$b=15.165(4) \AA$

$c=5.6079(11) \AA$

$\beta=91.41(2)^{\circ}$

$V=1009.3(4) \AA^{3}$

$Z=4$

$F(000)=416$

$D_{\mathrm{x}}=1.278 \mathrm{Mg} \mathrm{m}^{-3}$

Mo $K \alpha$ radiation, $\lambda=0.71073 \AA$

Cell parameters from 403 reflections

$\theta=4.3-21.1^{\circ}$

$\mu=0.09 \mathrm{~mm}^{-1}$

$T=100 \mathrm{~K}$

Needle, colourless

$0.20 \times 0.08 \times 0.06 \mathrm{~mm}$

Data collection

Agilent Xcalibur Sapphire3 diffractometer

Radiation source: Enhance (Mo) X-ray Source

Detector resolution: 16.1827 pixels $\mathrm{mm}^{-1}$

$\omega$-scan

Absorption correction: multi-scan

(CrysAlis RED; Agilent, 2012)

$T_{\min }=0.357, T_{\max }=1.000$

5292 measured reflections

1761 independent reflections

922 reflections with $I>2 \sigma(I)$

$R_{\text {int }}=0.101$

$\theta_{\max }=25.0^{\circ}, \theta_{\min }=3.2^{\circ}$

$h=-14 \rightarrow 13$

$k=-17 \rightarrow 18$

$l=-5 \rightarrow 6$

Refinement

Refinement on $F^{2}$

Least-squares matrix: full

$R\left[F^{2}>2 \sigma\left(F^{2}\right)\right]=0.090$

$w R\left(F^{2}\right)=0.255$

$S=1.03$

1761 reflections

132 parameters

Primary atom site location: structure-invariant direct methods

Secondary atom site location: difference Fourier map

Hydrogen site location: difference Fourier map

0 restraints

$\mathrm{H}$ atoms treated by a mixture of independent and constrained refinement 
$w=1 /\left[\sigma^{2}\left(F_{\mathrm{o}}^{2}\right)+(0.1022 P)^{2}\right]$

where $P=\left(F_{\mathrm{o}}^{2}+2 F_{\mathrm{c}}^{2}\right) / 3$

$(\Delta / \sigma)_{\max }<0.001$

$$
\Delta \rho_{\max }=0.30 \text { e } \AA^{-3}
$$

\section{Special details}

Geometry. All esds (except the esd in the dihedral angle between two 1.s. planes) are estimated using the full covariance matrix. The cell esds are taken into account individually in the estimation of esds in distances, angles and torsion angles; correlations between esds in cell parameters are only used when they are defined by crystal symmetry. An approximate (isotropic) treatment of cell esds is used for estimating esds involving l.s. planes.

Fractional atomic coordinates and isotropic or equivalent isotropic displacement parameters $\left(\AA^{2}\right)$

\begin{tabular}{lllll}
\hline & $x$ & $y$ & $z$ & $U_{\text {iso }} / U_{\text {eq }}$ \\
\hline O1 & $0.8289(3)$ & $0.2748(2)$ & $0.4019(6)$ & $0.0456(10)$ \\
H1O & $0.845(4)$ & $0.252(4)$ & $0.534(10)$ & $0.068^{*}$ \\
O2 & $0.8740(3)$ & $0.4013(2)$ & $0.0966(6)$ & $0.0476(11)$ \\
O3 & $0.8375(3)$ & $0.5068(2)$ & $0.3667(6)$ & $0.0455(10)$ \\
C1 & $0.5984(5)$ & $0.3361(3)$ & $0.3003(9)$ & $0.0443(14)$ \\
H1 & 0.6314 & 0.3108 & 0.1638 & $0.053^{*}$ \\
C2 & $0.4827(5)$ & $0.3407(3)$ & $0.3113(9)$ & $0.0479(14)$ \\
H2 & 0.4368 & 0.3171 & 0.1856 & $0.057^{*}$ \\
C3 & $0.4332(5)$ & $0.3801(3)$ & $0.5079(9)$ & $0.0492(15)$ \\
H3 & 0.3535 & 0.3852 & 0.5142 & $0.059^{*}$ \\
C4 & $0.4998(5)$ & $0.4111(3)$ & $0.6910(9)$ & $0.0467(14)$ \\
H4 & 0.4658 & 0.4367 & 0.8262 & $0.056^{*}$ \\
C5 & $0.6154(5)$ & $0.4061(3)$ & $0.6831(9)$ & $0.0419(13)$ \\
H5 & 0.6600 & 0.4286 & 0.8122 & $0.050^{*}$ \\
C6 & $0.6684(4)$ & $0.3679(3)$ & $0.4857(8)$ & $0.0387(13)$ \\
C7 & $0.7937(4)$ & $0.3603(3)$ & $0.4744(8)$ & $0.0403(13)$ \\
H7 & 0.8283 & 0.3743 & 0.6343 & $0.048^{*}$ \\
C8 & $0.8399(4)$ & $0.4230(3)$ & $0.2895(9)$ & $0.0416(13)$ \\
C9 & $0.8869(5)$ & $0.5744(3)$ & $0.2113(9)$ & $0.0452(14)$ \\
H9 & 0.9599 & 0.5521 & 0.1494 & $0.054^{*}$ \\
C10 & $0.8087(5)$ & $0.5957(4)$ & $0.0050(9)$ & $0.0565(16)$ \\
H10A & 0.7346 & 0.6120 & 0.0649 & $0.085^{*}$ \\
H10B & 0.8009 & 0.5440 & -0.0989 & $0.085^{*}$ \\
H10C & 0.8394 & 0.6451 & -0.0856 & $0.085^{*}$ \\
C11 & $0.9094(5)$ & $0.6527(3)$ & $0.3724(9)$ & $0.0528(15)$ \\
H11A & 0.9489 & 0.6329 & 0.5183 & $0.079^{*}$ \\
H11B & 0.8378 & 0.6802 & 0.4136 & $0.079^{*}$ \\
H11C & 0.9562 & 0.6956 & 0.2897 & $0.079^{*}$ \\
& & & &
\end{tabular}

Atomic displacement parameters $\left(\AA^{2}\right)$

\begin{tabular}{lllllll}
\hline & $U^{11}$ & $U^{22}$ & $U^{33}$ & $U^{12}$ & $U^{13}$ & $U^{23}$ \\
\hline O1 & $0.074(3)$ & $0.030(2)$ & $0.0327(19)$ & $0.0060(18)$ & $0.0021(18)$ & $0.0017(15)$ \\
O2 & $0.074(3)$ & $0.040(2)$ & $0.0298(19)$ & $-0.0036(18)$ & $0.0078(18)$ & $-0.0039(15)$ \\
O3 & $0.068(3)$ & $0.035(2)$ & $0.0337(19)$ & $-0.0031(18)$ & $0.0107(17)$ & $-0.0007(15)$ \\
C1 & $0.062(4)$ & $0.039(3)$ & $0.031(3)$ & $0.000(3)$ & $-0.003(2)$ & $0.003(2)$
\end{tabular}




\begin{tabular}{lllllll} 
C2 & $0.064(4)$ & $0.042(3)$ & $0.038(3)$ & $-0.001(3)$ & $-0.003(3)$ & $0.005(2)$ \\
C3 & $0.055(4)$ & $0.046(3)$ & $0.047(3)$ & $-0.001(3)$ & $-0.001(3)$ & $0.009(3)$ \\
C4 & $0.063(4)$ & $0.036(3)$ & $0.041(3)$ & $0.004(3)$ & $0.010(3)$ & $0.001(2)$ \\
C5 & $0.065(4)$ & $0.027(3)$ & $0.033(3)$ & $-0.004(3)$ & $0.001(2)$ & $0.003(2)$ \\
C6 & $0.055(4)$ & $0.033(3)$ & $0.029(3)$ & $-0.004(2)$ & $0.003(2)$ & $0.003(2)$ \\
C7 & $0.060(4)$ & $0.031(3)$ & $0.030(3)$ & $-0.001(3)$ & $0.003(2)$ & $-0.004(2)$ \\
C8 & $0.055(4)$ & $0.034(3)$ & $0.036(3)$ & $-0.002(3)$ & $-0.003(2)$ & $-0.002(2)$ \\
C9 & $0.061(4)$ & $0.038(3)$ & $0.037(3)$ & $-0.006(3)$ & $0.012(2)$ & $0.001(2)$ \\
C10 & $0.081(4)$ & $0.051(4)$ & $0.038(3)$ & $-0.015(3)$ & $0.001(3)$ & $0.010(3)$ \\
C11 & $0.074(4)$ & $0.039(3)$ & $0.045(3)$ & $-0.008(3)$ & $0.002(3)$ & $-0.001(2)$ \\
\hline
\end{tabular}

Geometric parameters $\left(A,{ }^{\circ}\right)$

\begin{tabular}{|c|c|c|c|}
\hline $\mathrm{O} 1-\mathrm{C} 7$ & $1.424(6)$ & $\mathrm{C} 3-\mathrm{C} 4$ & $1.364(8)$ \\
\hline $\mathrm{O} 2-\mathrm{C} 8$ & $1.211(5)$ & $\mathrm{C} 4-\mathrm{C} 5$ & $1.376(8)$ \\
\hline $\mathrm{O} 3-\mathrm{C} 8$ & $1.343(6)$ & $\mathrm{C} 5-\mathrm{C} 6$ & $1.412(7)$ \\
\hline $\mathrm{O} 3-\mathrm{C} 9$ & $1.476(6)$ & $\mathrm{C} 6-\mathrm{C} 7$ & $1.495(7)$ \\
\hline $\mathrm{C} 1-\mathrm{C} 2$ & $1.378(7)$ & $\mathrm{C} 7-\mathrm{C} 8$ & $1.519(7)$ \\
\hline $\mathrm{C} 1-\mathrm{C} 6$ & $1.401(7)$ & $\mathrm{C} 9-\mathrm{C} 10$ & $1.501(8)$ \\
\hline $\mathrm{C} 2-\mathrm{C} 3$ & $1.397(7)$ & $\mathrm{C} 9-\mathrm{C} 11$ & $1.512(7)$ \\
\hline $\mathrm{C} 8-\mathrm{O} 3-\mathrm{C} 9$ & $117.1(4)$ & $\mathrm{O} 1-\mathrm{C} 7-\mathrm{C} 6$ & $112.4(4)$ \\
\hline $\mathrm{C} 2-\mathrm{C} 1-\mathrm{C} 6$ & $121.6(5)$ & $\mathrm{O} 1-\mathrm{C} 7-\mathrm{C} 8$ & $105.2(4)$ \\
\hline $\mathrm{C} 1-\mathrm{C} 2-\mathrm{C} 3$ & $119.8(5)$ & $\mathrm{C} 6-\mathrm{C} 7-\mathrm{C} 8$ & $110.9(4)$ \\
\hline $\mathrm{C} 4-\mathrm{C} 3-\mathrm{C} 2$ & $119.6(6)$ & $\mathrm{O} 2-\mathrm{C} 8-\mathrm{O} 3$ & $123.8(5)$ \\
\hline $\mathrm{C} 3-\mathrm{C} 4-\mathrm{C} 5$ & $121.1(5)$ & $\mathrm{O} 2-\mathrm{C} 8-\mathrm{C} 7$ & $125.0(4)$ \\
\hline $\mathrm{C} 4-\mathrm{C} 5-\mathrm{C} 6$ & $120.8(5)$ & $\mathrm{O} 3-\mathrm{C} 8-\mathrm{C} 7$ & $111.2(4)$ \\
\hline $\mathrm{C} 1-\mathrm{C} 6-\mathrm{C} 5$ & $117.0(5)$ & $\mathrm{O} 3-\mathrm{C} 9-\mathrm{C} 10$ & $111.0(4)$ \\
\hline $\mathrm{C} 1-\mathrm{C} 6-\mathrm{C} 7$ & $121.0(5)$ & $\mathrm{O} 3-\mathrm{C} 9-\mathrm{C} 11$ & $105.0(4)$ \\
\hline $\mathrm{C} 5-\mathrm{C} 6-\mathrm{C} 7$ & $121.9(5)$ & $\mathrm{C} 10-\mathrm{C} 9-\mathrm{C} 11$ & $112.9(5)$ \\
\hline $\mathrm{C} 6-\mathrm{C} 1-\mathrm{C} 2-\mathrm{C} 3$ & $1.9(8)$ & $\mathrm{C} 1-\mathrm{C} 6-\mathrm{C} 7-\mathrm{C} 8$ & $71.2(6)$ \\
\hline $\mathrm{C} 1-\mathrm{C} 2-\mathrm{C} 3-\mathrm{C} 4$ & $-2.1(8)$ & $\mathrm{C} 5-\mathrm{C} 6-\mathrm{C} 7-\mathrm{C} 8$ & $-109.5(5)$ \\
\hline $\mathrm{C} 2-\mathrm{C} 3-\mathrm{C} 4-\mathrm{C} 5$ & $1.4(8)$ & $\mathrm{C} 9-\mathrm{O} 3-\mathrm{C} 8-\mathrm{O} 2$ & $-4.3(8)$ \\
\hline $\mathrm{C} 3-\mathrm{C} 4-\mathrm{C} 5-\mathrm{C} 6$ & $-0.5(7)$ & $\mathrm{C} 9-\mathrm{O} 3-\mathrm{C} 8-\mathrm{C} 7$ & $176.1(4)$ \\
\hline $\mathrm{C} 2-\mathrm{C} 1-\mathrm{C} 6-\mathrm{C} 5$ & $-0.9(7)$ & $\mathrm{O} 1-\mathrm{C} 7-\mathrm{C} 8-\mathrm{O} 2$ & $15.9(7)$ \\
\hline $\mathrm{C} 2-\mathrm{C} 1-\mathrm{C} 6-\mathrm{C} 7$ & $178.4(5)$ & $\mathrm{C} 6-\mathrm{C} 7-\mathrm{C} 8-\mathrm{O} 2$ & $-105.9(6)$ \\
\hline $\mathrm{C} 4-\mathrm{C} 5-\mathrm{C} 6-\mathrm{C} 1$ & $0.2(7)$ & $\mathrm{O} 1-\mathrm{C} 7-\mathrm{C} 8-\mathrm{O} 3$ & $-164.5(4)$ \\
\hline $\mathrm{C} 4-\mathrm{C} 5-\mathrm{C} 6-\mathrm{C} 7$ & $-179.1(4)$ & $\mathrm{C} 6-\mathrm{C} 7-\mathrm{C} 8-\mathrm{O} 3$ & $73.7(5)$ \\
\hline $\mathrm{C} 1-\mathrm{C} 6-\mathrm{C} 7-\mathrm{O} 1$ & $-46.2(6)$ & $\mathrm{C} 8-\mathrm{O} 3-\mathrm{C} 9-\mathrm{C} 10$ & $76.8(6)$ \\
\hline $\mathrm{C} 5-\mathrm{C} 6-\mathrm{C} 7-\mathrm{O} 1$ & $133.0(5)$ & $\mathrm{C} 8-\mathrm{O} 3-\mathrm{C} 9-\mathrm{C} 11$ & $-160.9(5)$ \\
\hline
\end{tabular}

Hydrogen-bond geometry $\left(\AA,{ }^{\circ}\right)$

\begin{tabular}{lllll}
\hline$D-\mathrm{H} \cdots A$ & $D-\mathrm{H}$ & $\mathrm{H} \cdots A$ & $D \cdots A$ & $D-\mathrm{H} \cdots A$ \\
\hline $\mathrm{O} 1-\mathrm{H} 1 O \cdots \mathrm{O}^{\mathrm{i}}$ & $0.83(6)$ & $2.12(5)$ & $2.903(2)$ & $158(5)$
\end{tabular}


supporting information

$\begin{array}{lllll}\mathrm{O} 1-\mathrm{H} 1 O \cdots \mathrm{O} 2^{\mathrm{i}} & 0.83(6) & 2.38(6) & 2.930(5) & 124(5) \\ \mathrm{C} 9-\mathrm{H} 9 \cdots \mathrm{O} 2^{\mathrm{ii}} & 1.00 & 2.53 & 3.379(7) & 142\end{array}$

Symmetry codes: (i) $x,-y+1 / 2, z+1 / 2$; (ii) $-x+2,-y+1,-z$. 